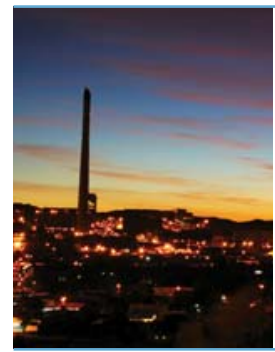

\title{
Acute respiratory infections among Indigenous children
}

\section{Population measures and getting the clinical basics right will help this complex and persistent problem}

T he extraordinarily high attack rates for acute respiratory infections among Indigenous children from remote areas of Australia are well known, ${ }^{1}$ but few studies have been conducted outside these areas. The Mount Isa-based study in this issue of the Journal ${ }^{2}$ is welcome, as it is the first on the burden of hospitalisation for acute respiratory infection in the north-west Queensland region. Although the study compared data on acute respiratory infections and bronchiectasis rates with other regions, the dissimilar methodology makes direct comparison problematic, particularly for pneumonia (with the lack of a gold standard for diagnosis ${ }^{3}$ ) and bronchiectasis (which is dependent on chest computed tomography criteria, and hence clinical vigilance and referral rates). Notwithstanding the limitations of the data, the article brings to the fore several issues.

While malnutrition, diarrhoeal disease and child mortality have all markedly declined among Indigenous children over the past 50 years, rates of acute respiratory infection have not, and short- and long-term morbidity rates remain substantially higher than for non-Indigenous children. ${ }^{4}$ In addition to the burden of hospitalisation, cost and social dislocation, it is likely that infectious insults to the developing lung impair subsequent lung growth and function. Unlike some other organs, the lung continues to grow until at least 7 to 8 years of age, while pulmonary immunity and respiratory disease phenotype are influenced by geneticenvironmental interaction that commences very early in life (possibly in utero). So it is highly plausible that many common conditions in adulthood emerge from an early childhood genesis. ${ }^{5}$ Indeed, there is increasing evidence that a substantial proportion of lung disease in adults, such as chronic obstructive lung disease and bronchiectasis, has its origins in childhood, when disease is both treatable and potentially reversible. ${ }^{5,6}$ In situations where management is suboptimal, Indigenous adults with bronchiectasis die in their third or fourth decade, ${ }^{7}$ whereas in optimal management situations, lung function in Australian children with bronchiectasis is stable or even improves. ${ }^{6}$

National data for Indigenous Australians show that respiratory disorders are the most common reason for general practice encounters, the second most prevalent self-reported chronic condition and the second most common cause for hospitalisation ${ }^{4}$ Overall, 27\% of Indigenous people report some form of respiratory disease $-19 \%$ in those aged $<14$ years and $38 \%$ in those aged $>55$ years. ${ }^{4}$ Also, acute respiratory infection with chronic cough is an independent risk factor for cardiovascular health. ${ }^{8}$

All this has implications for the importance of vaccine policy, standard treatment protocols, training of clinical staff and provision of clinical services. No one doubts that social determinants are of major importance in population health status, but the effects are extremely complex. This may explain why a systematic overview of interventions addressing the social determinants of health found a striking lack of reliable evaluations; and where evidence was available, the health improvement associated with interventions was modest or uncertain. ${ }^{9}$ However, we can make substantial gains in health for Indigenous children now. Clinicians and a competent primary health care service that efficiently links with the hospital system are vital. We need to get the basics right: high levels of vaccination coverage, and systems and training to ensure implementation of standard treatment protocols and early identification of children at risk of chronic suppurative lung disease, especially since chronic cough is often considered normal in communities where respiratory infections are common.

There are, of course, key population health actions, particularly antismoking strategies. Since poor neonatal and infant outcomes are risk factors for future chronic lung
Paul J Torzillo MBBS, FRACP, FCICM Executive Clinical Director and Medical Director ${ }^{2}$

Anne B Chang MBBS, PhD, FRACP Professor $^{3}$

1 Department of Respiratory Medicine and Department of Intensive Care, Royal Prince Alfred Hospital, Sydney, NSW.

2 Nganampa Health Council, Alice Springs, NT.

3 Queensland Children's Respiratory Centre, Royal Children's Hospital, Brisbane, QLD. paul.torzillo@ sydney.edu.au

doi: 10.5694/mjal4.00649 
diseases, ${ }^{5,10}$ attention to perinatal and early childhood care is key. Improved parenting is likely to be crucial, particularly in environments with many hazards for the health of infants and unborn babies. Given this complexity, the implementation in three Aboriginal health services of the nurse home-visiting model developed by Olds ${ }^{11}$ is an important step.

While Indigenous children who live in rural and remote regions provide particular challenges for the delivery of care, we need to maintain the objective of delivering bestpractice care in these locations.

Although there has been a significant closing of the health gap between Indigenous and non-Indigenous people between 1991 and 2010, ${ }^{12}$ the gap is still large. Changes in social indicators are important not just for health but for social justice; however, for improving child health in particular, both clinical medicine and population health measures still have a key role.

Acknowledgements: Anne Chang is supported by a National Health and Medical Research Council Practitioner Fellowship (1058213).

Competing interests: No relevant disclosures.

Provenance: Commissioned; not externally peer reviewed.

1 O'Grady KA, Taylor-Thomson D, Chang AB, et al. Rates of World Health Organization-defined radiologically confirmed pneumonia in Northern Territory Indigenous children. Med J Aust 2010; 192: 592-595.
2 Janu EK, Annabattula BI, Kumariah S, et al. Paediatric hospitalisations for lower respiratory tract infections in Mount Isa. Med J Aust 2014; 200 591-594.

3 Chang AB, Ooi MH, Perera D, Grimwood K. Improving the diagnosis, management and outcomes of children with pneumonia: where are the gaps? Front Pediatr 2013; 1: 29. doi: 10.3389/fped.2013.00029.

4 Australian Institute of Health and Welfare. The health and welfare of Australia's Aboriginal and Torres Strait Islander people: an overview 2011. Canberra: AlHW, 2011. (AlHW Cat. No. IHW 42.)

5 Stocks J, Hislop A, Sonnappa S. Early lung development: lifelong effect on respiratory health and disease. Lancet Respir Med 2013; 1: 728-742.

6 Chang AB, Byrnes CA, Everard ML. Diagnosing and preventing chronic suppurative lung disease (CSLD) and bronchiectasis. Paediatr Respir Rev 2011; 12: 97-103.

7 Steinfort DP, Brady S, Weisinger HS, Einseidel L. Bronchiectasis in Central Australia: a young face to an old disease. Respir Med 2008; 102: 574-578.

8 Kiechl S, Egger G, Mayr M, Weidermann CJ, et al. Chronic infection and the risk of carotid atherosclerosis: prospective results from a large population study. Circulation 2001; 103: 1064-1070.

9 Petticrew M, Bambra C, Gibson M, et al. Tackling the wider social determinants of health and health inequalities: evidence from systematic reviews. Final report. Public Health Research Consortium, 2009. http:// phrc.Ishtm.ac.uk/project_2005-2011_d206.html (accessed May 2014).

10 Singleton RJ, Valery PC, Morris P, et al. Indigenous children from three countries with non-cystic fibrosis chronic suppurative lung disease/ bronchiectasis. Pediatr Pulmonol 2014; 49: 189-200.

11 Olds DL. Prenatal and infancy home visiting by nurses: from randomized trials to community replication. Prev Sci 2002; 3: 153-172.

12 Australian Health Ministers' Advisory Council. Aboriginal and Torres Strait Islander Health Performance Framework 2012 Report. Canberra: AHMAC, 2012. 